\title{
Le syndrome de Coffin-Lowry : une anomalie de la transduction du signal (voie Ras/MAP kinase)
}

Le syndrome de Coffin-Lowry (CLS) est une maladie malformative très sévère, associant notamment un retard mental important et des déformations squelettiques progressives $[1,2]$. Il s'agit d'une maladie liée au chromosome X (les garçons étant sévèrement atteints, les filles hétérozygotes manifestant, en général, des signes discrets de la maladie). La maladie paraît rare, mais de nombreux cas ne sont probablement pas diagnostiqués. Notre équipe avait effectué, en 1988, la première localisation du gène de cette maladie (région Xp22) [3]. A partir de 1991, une recherche systématique de familles, au plan international, nous a permis d'affiner considérablement la localisation (dans un intervalle de 3-4 centimorgans) $[4,5]$. Nous avons entrepris la construction d'une carte physique et transcriptionnelle de cette région. L'un des gènes ainsi caractérisés a été retrouvé muté dans six familles avec CLS [6]. Ce gène code pour une protéine kinase de la famille RSK (ou p90 ${ }^{R S K}$ ), qui comprend trois membres chez l'homme (RSK1, 2 et 3) [7]. Il s'agit de sérinethréonine kinases, impliquées dans la voie de réponse aux agents mitogènes (cascade Ras/MAP kinases), agissant sur la prolifération et la différenciation cellulaires (avec des cibles telles que les facteurs de transcription Fos, Jun, Nur77 et CREB) $\left(\mathrm{m} / \mathrm{s} n^{\circ} 11\right.$, vol. 12, p. 1258) [8, 9]. Jusqu'à présent, il n'était pas possible de différencier les contributions fonctionnelles respectives des trois protéines RSK. L'implication du gène $R S K 2$ dans le syndrome de Coffin-Lowry ouvre donc de nouvelles perspectives d'étude de cette voie fondamentale de transduction du signal (recherche des signaux d'activation en amont, des cibles spécifiques en aval). Les premières mutations mises en évidence (jusqu'à présent) correspondent, soit à des pertes d'expression de la protéine RSK2, soit, de manière très intéressante, à des faux-sens susceptibles d'altérer des domaines fonctionnels importants de cette dernière.

En ce qui concerne la maladie, la recherche des mutations permettra un meilleur diagnostic et un meilleur conseil génétique. Dans la plupart des familles, il s'agit de cas sporadiques; il était donc très difficile d'estimer le risque de récurrence, ou de préciser le statut des femmes à risque d'être vectrices. Comme pour les autres maladies sévères liées à $\mathrm{l}$ 'X, on s'attend a priori à une très grande hétérogénéité des mutations (une mutation différente par famille), ce qui n'est pas sans poser des problèmes quant à la mise en ouvre des applications diagnostiques. La découverte d'une mutation faux-sens chez un patient atteint d'une forme nettement moins sévère de la maladie suggère que celle-ci recouvre un spectre clinique plus étendu que ce qui était rapporté jusqu'à présent (formes avec retard mental et dysmorphie plus modérés).

Rappelons aussi que, très récemment, le laboratoire de Marc Montminy (Boston, MA, USA) a montré que l'activation de $\mathrm{p} 90^{\text {rk }}$ par divers facteurs (insuline, NGF) provoque la formation d'un complexe avec la protéine CBP/p300 qui semble indispensable aux effets différenciateurs du NGF sur des cellules chromaffines/neuronales PC12 en culture $\left(\mathrm{m} / \mathrm{s} n^{\circ} 11\right.$, vol. 12, p. 1258). Or, le gène $C B P$ est muté de façon hétérozygote chez les malades atteints de syndrome de Rubinstein-Taybi, caractérisé par un tableau dysmorphique et un retard mental $\left(\mathrm{m} / \mathrm{s} n^{\circ} 10\right.$, vol. 11, p. 1496). Quoique les signes des maladies de Coffin-Lowry et de Rubinstein-Taybi ne soient pas semblables, le retard mental de ces deux maladies est-il lié aux anomalies d'une voie de transmission d'un signal relayé, dans le système nerveux central, par le complexe $\mathrm{p} 90^{R S K} / \mathrm{CBP}$ ?

\section{André Hanauer \\ Élisabeth Trivier \\ Dario De Cesare \\ Sylvie Jacquot \\ Solange Pannetier \\ Paolo Sassone-Corsi \\ Jean-Louis Mandel}

Institut de génétique et de biologie moléculaire et cellulaire, Cnrs/Inserm/Ulp, BP 163, 67404 Illkirch Cedex, France.

1. Young ID, The Coffin-Lowry syndrome. J Med Genet 1988; 25 : 344-8.

2. Gilgenkrantz S, Mujica P, Gruet P, Tridon P, Schweitzer F, Nivelon-Chevalier A, Nivelon JL, Couillault G, David A, Verloes A, Lambotte C, Piussan C, Mathieu M. Coffin-Lowry syndrome: a multicenter study. Clin Genet 1988; $34: 230$ 45.

3. Hanauer A, Alembik Y, Gilgenkrantz S, Mujica P, Nivelon-Chevalier A, Pembrey ME, Young ID, Mandel JL. Probable localisation of the Coffin-Lowry locus in Xp22.2-p22.1 by multipoint linkage analysis. Am J Med Genet 1988; 30 : 523-30. 
4. Biancalana V, Briard ML, David A, Gilgenkrantz S, Kaplan J, Mathieu M, Piussan C, Poncin J, Schinzel A, Oudet C, Hanauer A. Confirmation and refinement of the genetic localization of the Coffin-Lowry syndrome locus in Xp22.1-p22.2. Am J Hum Genet 1992; 50: 981-7.

5. Biancalana V, Trivier E, Weber C, Weissenbach J, Rowe PSN, O'Riordan JLH, Partington MW, Heyberger S, Oudet C, Hanauer A. Construction of a high-resolution linkage map for Xp22.1-p22.2 and refinement of the genetic localization of the Coffin-Lowry syndrome gene. Genomics 1994 ; 22 617-25.

6. Trivier E, De Cesare D, Jacquot S, Pannetier S, Zackai E, Young I, Mandel JL, Sassone-Corsi P, Hanauer A. Coffin-Lowry syndrome is caused by mutations in the growth-factor activated RSK2 kinase. Nature $1996 ; 384$ : 567-70.

7. Moller DE, Xia CH, Tang T, Zhu A, Jakubowsky
M. Human RSK isoforms: cloning and characterization of tissue-specific expression. Am J Physiol 1994; 266 : C351-9.

8. Blenis J. Signal transduction via the MAP kinases : proceed at your own RSK. Proc Natl Acad Sci USA 1993; 90 : 5889-92.

9. Xing J, Ginty DD, Greenberg ME. Coupling of the RAS-MAPK pathway to gene activation by RSK2, a growth factor-regulated CREB kinase Science 1996; 273: 959-63.

\section{BRÈVES}

Effets toxiques de l'oxygène, maladie d'Alzheimer et apolipoprotéine E. Nous avons rapporté très récemment que le peptide $\beta$-amyloïde pouvait se lier à des récepteurs membranaires et entraîner un stress oxydatif avec production de radicaux libres de l'oxygène $(\mathrm{m} / \mathrm{s}$ $n^{\circ} 11$, vol. 12, p. 1262). Par ailleurs, la corrélation entre la susceptibilité à la maladie d'Alzheimer et les allèles de l'apolipoprotéine E exprimés est connue depuis plusieurs années $\left(m / s n^{\circ} 10\right.$, vol. $9, p$. 1142). L'apolipoprotéine E2 semble être plutôt protectrice $\left(m / s n^{\circ} 8 / 9, \mathrm{vol}\right.$. $10, p$. 920) alors que l'apolipoprotéine E4 est clairement un important facteur de susceptibilité $(\mathrm{m} / \mathrm{s}$ $n^{\circ} 4$, vol. 10, p. 470). Par conséquent, il était logique de se poser la question d'un éventuel effet protecteur des allèles de l'apolipoprotéine E vis-à-vis des lésions oxydatives. Miyata et Smith, de New York (USA), ont spécifiquement étudié cette question [1]. Ils montrent que les allèles de cette apolipoprotéine sont, en effet, plus ou moins efficaces pour protéger les cellules des lésions provoquées par l'ajout d'eau oxygénée. L'effet protecteur est maximal pour l'allèle E2, moindre pour E3 et minimal pour E4 [1]. Le même effet est retrouvé en ce qui concerne la protection contre la cytotoxicité du peptide $\beta$-amyloïde. Par ailleurs, il est montré que les apolipoprotéines E sont capables de fixer des cations métalliques $\left(\mathrm{Cu}^{2+}\right.$, $\left.\mathrm{Fe}^{3+}, \mathrm{Fe}^{2+}, \mathrm{Zn}^{2+}\right)$, ce qui pourrait rendre compte de leur effet antioxydant. Cependant, aucun des auteurs n'a pu démontrer de différence dans l'affinité des allèles de
l'apoE pour ces cations métalliques. Naturellement, cette étude n'établit qu'une corrélation entre l'effet antioxydant des allèles de l'apoE et leur effet plus ou moins protecteur vis-àvis du développement de la maladie d'Alzheimer, sans démontrer le lien de cause à effet. Cependant, les arguments convergents en faveur de l'intervention de mécanismes de toxicité oxydative dans les lésions de la maladie d'Alzheimer rendent cette corrélation extrêmement suggestive.

[1. Miyata M, Smith JD. Nature Genet 1996 ; 14: 55-61.]

Rythmes et émotions sous la coupe des récepteurs histaminiques $\mathrm{Hl}$ ? Distinguer le rôle propre d'un récepteur lorsqu'il en existe différents sous-types et élucider sa fonction spécifique au niveau du système nerveux central sont deux objectifs particulièrement ambitieux. Cela est devenu possible grâce à la stratégie d'invalidation génique, comme le montre l'exemple du récepteur $\mathrm{Hl}$ de l'histamine [1]. Outre son rôle dans l'inflammation et la sécrétion gastrique acide, l'histamine, du fait de sa localisation neuronale cérébrale, exerce un rôle modulateur important au niveau du système nerveux central. Aujourd'hui, en invalidant chez la souris le gène codant pour le récepteur $\mathrm{Hl}$, un des trois sous-types de récepteurs histaminiques connus [2], un rapport japonais nous révèle l'importance de ce récepteur sur le comportement, $\operatorname{tant}$ au niveau de l'activité locomotrice que de l'émotivité. Si les souris dépourvues de récepteurs $\mathrm{Hl}$ n'ont aucune anomalie anatomique détectable, elles présentent, en revanche, une perturbation importante du rythme de leur activité locomotrice: au cours du cycle jour/nuit classique (12 heures/12 heures), ont été enregistrées à la fois une nette augmentation de l'activité locomotrice dans la période jour, et une diminution durant la phase nocturne. En outre, le rapport activité diurne/activité nocturne, comparé à celui des souris témoins, devient plus important chez les souris mutées. L'émotivité des souris est, elle aussi, mise à l'épreuve par l'invalidation du gène du récepteur H1. Dans une situation de stress, les signes émotionnels (miction, défécation, immobilisation) sont amplifiés chez les souris mutées. Placées dans un nouvel environnement, leur comportement explorateur est profondément altéré: alors que les mouvements moteurs réflexes restent coordonnés, la surface explorée et les temps d'exploration s'avèrent sensiblement diminués. L'ensemble de ces résultats mettent en lumière l'influence du système histaminergique cérébral relayé par les récepteurs histaminiques $\mathrm{Hl}$ sur le rythme jour/nuit des activités locomotrices et sur le contrôle émotionnel, deux composantes essentielles du comportement.

[1. Inoue I, et al. Proc Natl Acad Sci USA 1996; 93: 13316-20.]

[2. Devillier P, et al. Med Sci 1989; 5 : 408-14.] 